TI 2008-120/1

Tinbergen Institute Discussion Paper

\title{
Maximal Cartel Pricing and Leniency Programs
}

\author{
Harold Houba',2 \\ Evgenia Motchenkova' \\ Quan Wen ${ }^{3}$
}

\footnotetext{
${ }^{\prime}$ VU University Amsterdam;

2 Tinbergen Institute;

3 Vanderbilt University.
} 


\section{Tinbergen Institute}

The Tinbergen Institute is the institute for economic research of the Erasmus Universiteit Rotterdam, Universiteit van Amsterdam, and Vrije Universiteit Amsterdam.

Tinbergen Institute Amsterdam

Roetersstraat 31

1018 WB Amsterdam

The Netherlands

Tel.: +31(0)205513500

Fax: $+31(0) 205513555$

Tinbergen Institute Rotterdam

Burg. Oudlaan 50

3062 PA Rotterdam

The Netherlands

Tel.: + $31(0) 104088900$

Fax: $+31(0) 104089031$

Most TI discussion papers can be downloaded at http://www.tinbergen.nl. 


\title{
Maximal cartel pricing and leniency programs*
}

\author{
Harold Houba ${ }^{\dagger}$ \\ VU University Amsterdam \\ and Tinbergen Institute
}

\author{
Evgenia Motchenkova ${ }^{\ddagger}$ \\ VU University Amsterdam
}

Quan Wen ${ }^{\S}$

Vanderbilt University

December 2008

\begin{abstract}
For a general class of oligopoly models with price competition, we analyze the impact of ex-ante leniency programs in antitrust regulation on the endogenous maximalsustainable cartel price. This impact depends upon industry characteristics including its cartel culture. Our analysis disentangles the effects of traditional antitrust regulation and the leniency program. Ex-ante leniency programs are effective if and only if these offer substantial rewards to the self-reporting firm. This is in contrast to currently employed programs that are therefore ineffective.
\end{abstract}

JEL Classification: L41 Monopolization, Horizontal Anticompetitive Practices, K21 Antitrust Law, C72 Noncooperative Games.

Keywords: Cartel, Antitrust Policy, Antitrust Law, Leniency Program, Self-reporting, repeated game

*We would like to thank Els Kleijn who suggested to consider cartel cultures, Dave Furth who suggested to consider the homogeneous Bertrand oligopoly with linear demand and, finally Rene van den Brink, Arantza Estevez-Fernandez, Gerard van der Laan and Ines Lindner who suggested valuable contributions to an earlier draft.

†Department of Econometrics, VU University Amsterdam, De Boelelaan 1105, 1081 HV Amsterdam, Netherlands. Email: hhouba@feweb.vu.nl.

$\ddagger$ Department of Economics, VU University Amsterdam, De Boelelaan 1105, 1081 HV Amsterdam, Netherlands. Email: emotchenkova@feweb.vu.nl.

§Department of Economics, Vanderbilt University, VU Station B \#351819, 2301 Vanderbilt Place Nashville, TN 37235-1819, U.S.A. Email: quan.wen@vanderbilt.edu. 


\section{Introduction}

During the last two decades, antitrust policies in the US and the EC have undergone substantial reforms and currently include leniency programs as a key ingredient, e.g. US Department of Justice (1993) and EC (2002). Leniency programs grant total or partial immunity from fines to firms collaborating with the antitrust authority (AA) by revealing crucial information about the existence of the cartel that is needed for a successful conviction by penal courts. Information may be revealed ex-ante before an investigation conducted by the AA starts, or ex-post during an ongoing investigation. Leniency programs are based upon the economic principle that firms, who broke the antitrust law, might report their illegal activities if given proper incentives to do so and, if effective, might dissolve existing cartels or, even better, a priori deter such illegal activities. In the US, antitrust policy specifies (reduced) fines that are related to the cartel's net gain, and caught cartel members face additional liabilities in the form of private law suites by harmed customers. The EC legislation has penalty schemes for conviction and leniency that are proportional to the cartel's gain. Even though it is legally possible within many EU countries, private lawsuits are almost absent.

Despite a large literature on the theory of enforcement against individual illegal behavior, see e.g. Polinsky and Shavell (2000), the theory of antitrust policy is still in its infancy when it comes to enforcing market competition with little attention to its implementation, see e.g. Rey (2003) and Spagnolo (2007). Illegal anti-competitive behavior is a much more complicated subject since it typically is a concerted illegal action performed within an ongoing relationship over time, called a cartel. The theory of antitrust policy must therefore be conducted in a dynamic setting. The early literature deals with cartel stability in the absence of policy intervention, often modelled as a repeated game as in e.g. Abreu et al. (1986) and Green and Porter (1984). Recently, optimal implementation of antitrust policy

for cartel enforcement has been analyzed in e.g. Motta and Polo (2003), Spagnolo (2004, 2007), Harrington (2004, 2005), Hinloopen (2003, 2006), Motchenkova (2004), Buccirossi and Spagnolo (2006) and Harrington and Chen (2006). 
The seminal paper on optimal revelation schemes as part of antitrust policy is Motta and Polo (2003), who study, loosely speaking, a Stackelberg game in which the AA first chooses once and for all its antitrust policy followed by the competition phase in which the firms compete among each other, which is modelled as an infinitely-repeated oligopoly game. Market competition is restricted to a discrete set of three prices that captures the three most important profit levels: The profit under perfect competition, under the cartel and the profit of optimally cheating on the cartel price. In each period, firms decide whether to reveal information about their misconduct. The cartel adopts grim-trigger strategies in which cheating on the cartel by either setting a different price or applying for leniency triggers competitive behavior forever, while the cartel continues its illegal business as usual each time it is caught by the AA. Under the optimal antitrust policy, introduction of ex-post leniency programs will increase the chance of the cartel being captured, but ex-ante leniency programs that grant reduced fines are ineffective. As later shown in Spagnolo (2004) and Rey (2003), effective ex-ante leniency programs require substantial rewards, i.e., pay self-reporting firms.

Harrington and Chen (2006) incorporate ex-ante leniency programs into a special case of the framework in Harrington (2004 and 2005) to augment traditional antitrust policy in an environment where cartels arouse suspicions. In such environment, cartels also need to manage suspicions, modeled as if the cartel keeps in mind an endogenous detection probability. This probability is modelled as a function of the cartel's price setting, where price is a continuous variable. The focus is on an exogenous antitrust policy in order to study the cartel's optimal reaction on the profit-maximizing cartel price, which means that the price setting by the cartel has become an endogenous decision. This model can be regarded as the competition phase in Motta and Polo (2003) in a very general setting. The cartel adopts grim-trigger strategies that are similar to those in Motta and Polo (2003), but with the difference that the cartel terminates its illegal business after being caught once by the AA. So, the cartel culture of whether to continue the cartel after being caught differs between Motta and Polo (2003) and Harrington and Chen (2006). In the last reference, the detection prob- 
ability also depends upon past prices and collusive behavior induces a cumulative liability in the form of fixed fines and private law suites. These two features introduce state variables into the model and this makes the equilibrium nontractable. The analysis, therefore, has to resort to simulations of price paths. Nevertheless, the model admits a steady-state profitmaximizing cartel price that lies above the competitive price, and this price is independent of the leniency program meaning such program is ineffective.

The foci of our paper are also detection probabilities and penalty schemes that are sensitive to the cartel's pricing behavior within a generalized repeated-game version of Harrington and Chen (2006). Our model generalizes Motta and Polo (2003) by including endogenous cartel behavior, the presence of suspicions, the notion of cartel culture ${ }^{1}$, and a general class of exogenous antitrust policies with proportional penalty schemes that include the possibility of an effective reward. Several aspects of the model in Harrington and Chen (2006) are also generalized, namely a general oligopoly model instead of Bertrand oligopoly, general penalty schemes with fine reductions that include rewards, and the introduction of cartel culture. In fact, our notion of cartel culture unifies the opposite assumptions in Motta and Polo (2003) and Harrington and Chen (2006) with respect to this parameter. Therefore, our model bridges the rudimentary market competition phase in Motta and Polo (2003) with the general approach in Harrington and Chen (2006).

An innovative but unconventional aspect of our model is that we analyze the maximal cartel price, i.e., the largest cartel price for which the equilibrium conditions for sustainability hold, instead of the standard approach of profit maximization by the cartel. There are several reasons why the maximal cartel price is interesting: First, experimental economics has established that often economic agents behave differently from standard microeconomic theory. This hints at that cartel members may also behave different from profit maximization. Indeed, there is empirical evidence in support of Baumol's hypothesis that managers of large

\footnotetext{
${ }^{1}$ Cartel culture is related to the probability that the cartel resumes business as usual after each conviction by the antitrust authorities. Bosch and Eckard (1991) report that 14 per cent of 1300 firms are recidivist. This implies, on average, a cartel culture of .86 in our model.
} 
corporations seek to maximize sales rather than profits, see e.g. Baumol (1958) and McGuire et al. (1962). As an alternative, the sustainability of cartel behavior offers a more robust criterion that does not depend on the cartel's objective. Furthermore, the characterization of society's maximal damage through the maximal cartel price can be regarded as a worst-case scenario for society's unawareness about the cartel's objective. Second, under traditional antitrust regulation, we report that the cartel's value function may fail to be monotonically increasing and concave in the cartel price for all parameter values. This poses a technical problem in characterizing the profit-maximizing cartel price. We show that the maximal cartel price does not have this drawback. Third, there is a large subclass of parameter values for which the equilibrium conditions are binding under both the profit-maximizing cartel price and the maximal cartel price and, hence, both cartel prices coincide. This includes the subclass of parameter values for which the value function is monotonic.

Our analysis shows that a direct approach to characterize the maximal cartel price based upon inverting the (possibly non-monotonic) value function is not applicable. To overcome this technical issue, our characterization is based upon analyzing properties of the threshold level for the discount factor as a function of price and, then, translate these properties into the maximal cartel price as a function of the discount factor. This novel approach turns out to be very fruitful to characterize the maximal cartel price. This price is increasing in the discount factor, and it decreases in the cartel culture parameter and in the parameters characterizing penalty schemes (the coefficients of proportional fines and detection probabilities).

For a general class of ex-ante leniency programs, we show that such programs cannot be effective without rewards. We also characterize the minimal effective reward that is needed to make ex-ante leniency programs effective and break the cartel. The minimal effective reward critically depends upon the traditional antitrust policy employed. With respect to analyzing leniency programs, we also make a technical but useful remark in checking and deriving equilibrium strategies, application of the one-stage-deviation principle implies that one does not need to investigate simultaneously a deviation in the price setting stage and 
the revelation stage.

Our characterization of the maximal cartel price under traditional antitrust policy complements the results in Harrington (2004 and 2005). In the latter references, it is shown that the profit-maximizing cartel price has non-binding equilibrium conditions for sufficiently large discount factors and that it is strictly decreasing in the discount factor. Combined with our results, monotonicity of the profit-maximizing cartel price fails for the entire range of discount factors, which we illustrate by means of a robust example. In this example, the profit-maximizing cartel price also underestimates by far the maximal damage to society whenever the equilibrium conditions are non-binding. Finally, our results also hint at that the ineffectiveness of ex-ante leniency programs without rewards, as reported in Harrington and Chen (2006) for very large discount factors, generalizes to all discount factors.

The paper is organized as follows. Section 2 outlines the benchmark model where we derive maximal cartel price in the absence of any regulation. In section 3 we analyze the impact of traditional tools of antitrust enforcement on maximal sustainable cartel price and relate it to the characteristics of the industry. In section 4 we compare the effectiveness of antitrust enforcement supplemented by leniency programs to the results of the previous section. Section 5 concludes the analysis and discusses the policy recommendations.

\section{The Benchmark Model}

Consider an oligopoly market where $n \geq 2$ firms compete over infinitely many periods. Assume that all firms have a common discount factor $\delta \in(0,1)$ per period. During each period, firms compete in a symmetric Bertrand model with either homogenous or heterogeneous products. Let $\pi(p)$ be an individual firm's profit function in any period with price profile $p \in \mathbb{R}_{+}^{n}$. Since we often deal with symmetric outcomes, we simplify $\pi(p, \ldots, p) \equiv \pi(p)$. Denote the static Nash equilibrium price and the maximal collusive price by $p^{N}$ and $p^{M}$, re-

spectively. To simplify the exposition, we normalize the static model so that $\pi\left(p^{N}\right)=0$. In every period, the firms decide whether to collude and if so, to what degree. In other 
words, all firms choose a price $p=p^{N}+I\left(p^{M}-p^{N}\right)$, where $I \in[0,1]$ can be considered as a collusion index. One key element in analyzing cartel stability is a firm's profit from unilateral deviation against the cartel when all the other firms set their prices at $p$, denoted as $\pi^{\text {opt }}(p)=\sup _{p^{\prime}} \pi\left(p^{\prime}, p, \ldots, p\right)$. We deliberately write supremum instead of the maximum in order to also have a well-defined value function for the discontinuous case of homogeneous products. In order to capture the relevant oligopoly models in the literature, we assume that $\pi(p)$ is continuous and strictly increasing for $p \in\left[p^{N}, p^{M}\right], \pi^{o p t}(p)$ is continuous and $\pi^{o p t}(p)>\pi(p)>0$ for $p \in\left(p^{N}, p^{M}\right] .^{2}$

A novelty is that we define $\lambda(p)$ as the relative size of the cartel profit to the profit under best unilateral deviation. More specifically,

$$
\lambda(p)= \begin{cases}\frac{\pi(p)}{\pi^{o p t}(p)}, & \text { for } p \in\left(p^{N}, p^{M}\right], \\ 1, & \text { for } p=p^{N} .\end{cases}
$$

Our assumptions on $\pi(p)$ imply that $\lambda(p)<\lambda\left(p^{N}\right) \equiv 1$ for all $p \in\left(p^{N}, p^{M}\right]$. Since the function $\lambda(\cdot)$ might be discontinuous at $p=p^{N}$, as Example 2 illustrates, we introduce ${ }^{3}$

$$
\underline{\lambda}=\lim _{\varepsilon \rightarrow 0^{+}} \lambda\left(p^{N}+\varepsilon\right) \leq 1 \quad \text { and } \quad \bar{\lambda}=\lambda\left(p^{M}\right) .
$$

To our analysis, two sectors with the same $\lambda(\cdot)$ function will be treated as identical. Note that $\lambda(\cdot)$ is the ratio of individual firm's profit from the cartel to the profit from unilateral optimal deviation against the cartel. This means that higher $\lambda(\cdot)$, the less incentives each cartel member has to deviate, and the more stable the cartel is. Consequently, $\lambda(\cdot)$ represents the degree of incentives to deviate or degree of cartel stability in the sector. We assume that $\lambda(p)$ is non-increasing for all $p \in\left(p^{N}, p^{M}\right]$, so that the higher the cartel price, the higher the incentive of each cartel member to deviate. ${ }^{4}$

We will analyze antitrust regulation and leniency programs in Sections 3 and 4, respectively. The underlying model with or without antitrust regulation is a standard infinitely-

\footnotetext{
${ }^{2}$ Note that this setup is identical to Harrington (2004 and 2005).

${ }^{3}$ The limit $\underline{\lambda}$ is well defined, because the correspondence $\hat{\lambda}(\cdot)$ that solves $\hat{\lambda}(p) \pi^{\text {opt }}(p)=\pi(p)$ for each $p \in\left[p^{N}, p^{M}\right]$ is upper semi-continuous for all $p \in\left[p^{N}, p^{M}\right]$ and it coincides with the continuous function $\lambda(\cdot)$ for all $p>p^{N}$.

${ }^{4}$ This is a mild assumption that captures the class of symmetric heterogeneous Bertrand duopolies with linear demand functions and common constant marginal costs.
} 
repeated game with the static model described above as the stage game. Under a leniency program, however, the model becomes an infinitely-repeated sequential game since firms may self-report to the AA. Throughout this paper, we focus on a class of modified grim-trigger strategies to sustain cartel price $p>p^{N}$ in which any deviation leads to the repetition of the perfectly competitive equilibrium outcome in every period thereafter. The underlying rationale is that cartels are based upon trust and, by the reciprocal nature of humans, all trust is gone after someone cheats.

In this paper, the equilibrium concept is subgame perfect equilibrium. In verifying equilibrium conditions, we apply the one-stage-deviation principle, see e.g., Fudenberg and Tirole (1991). This principle states that it is sufficient to consider only so-called one-stage deviations $^{5}$ instead of verifying all possible deviating strategies. Especially for the model with a leniency program, we only need to consider unilateral deviations at either the price setting stage or the self-reporting stage but not both stages simultaneously. To verify equilibrium conditions, only the expected profits from unilateral one-stage deviations are required.

In the remainder of this section, we analyze the benchmark in the absence of any antitrust regulation and leniency program under the standard grim-trigger strategy profile to sustain a cartel price of $p>p^{N}$. In the absence of any antitrust regulation, the necessary and sufficient condition to support $p \in\left(p^{N}, p^{M}\right]$ as a cartel price is

$$
\pi^{o p t}(p)+\frac{\delta}{1-\delta} \pi\left(p^{N}\right) \leq \frac{1}{1-\delta} \pi(p) \Longleftrightarrow \delta \geq 1-\lambda(p)
$$

Since $\lambda(p)$ is non-increasing in $p,(1)$ implies that the threshold for $\delta$ is non-decreasing in the cartel price $p \in\left(p^{N}, p^{M}\right]$, and it is equivalent to $p \leq \max \left\{p: p \in \lambda^{-1}(1-\delta)\right\}$. Because $p^{N}$ satisfies (1) for all $\delta \in(0,1)$, we have $p^{N} \leq \max \left\{p: p \in \lambda^{-1}(1-\delta)\right\}$. The socially worst outcome is the maximal cartel price supported by the grim-trigger strategy profiles; ${ }^{6}$

$$
p^{C}=\max _{p \in\left[p^{N}, p^{M}\right]} p, \quad \text { s.t. }(1) .
$$

\footnotetext{
${ }^{5}$ A one-stage deviation is a strategy that almost coincides with the strategy under consideration, except at one particular stage and history where it prescribes a different action.

${ }^{6}$ The idea of an endogenous cartel price is also studied in Harrington (2004).
} 
The maximal cartel price $p^{C}$ is well defined since (1) induces a closed subinterval (possibly degenerated) of $\left[p^{N}, p^{M}\right]$.

Due the monotonicity of $\pi(p)$, the profit of each cartel member is also maximized at the maximal cartel price $p^{C}$ among all subgame perfect equilibria supported by grim-trigger strategy profiles. However, as we will see later, this may not be the case under an antitrust regulation with or without a leniency program.

Now we explain how we characterize the maximal cartel price $p^{C}$ later on. A direct approach would be to solve (1) for $p$ as a function of all parameters, which requires the inverse function $\lambda^{-1}(1-\delta)$. In Section 3 and 4 , however, the function of $p$ that needs to be inverted becomes very complicated and may even lack monotonicity properties. Instead, our characterization is based upon analyzing properties of the threshold level for $\delta$ as a function of $p \in\left[p^{N}, p^{M}\right]$ and then, translate these properties to the maximal cartel price as a function of $\delta$ in the $(\delta, p)$-plane. This indirect approach turns out to be very fruitful, as the following result for the benchmark demonstrates.

Proposition 1 In the absence of any antitrust regulation, the maximal cartel price $p^{C}$ is non-decreasing in $\delta \in(0,1)$, and

$$
\begin{array}{ll}
p^{C}=p^{N}, & \text { for } \delta \in(0,1-\underline{\lambda}), \\
p^{C} \in\left[p^{N}, p^{M}\right), & \text { for } \delta \in[1-\underline{\lambda}, 1-\overline{\bar{\lambda}}), \\
p^{C}=p^{M}, & \text { for } \delta \in[1-\bar{\lambda}, 1) .
\end{array}
$$

Proof. For $\delta^{\prime}>\delta, \delta \geq 1-\lambda(p)$ implies that $\delta^{\prime} \geq 1-\lambda(p)$. This means that any $p \in\left[p^{N}, p^{M}\right]$ that satisfies (2) for $\delta$ must also satisfy (2) for $\delta^{\prime}$. Hence, $p^{C}$ must be nondecreasing in $\delta \in(0,1)$. If $\delta<1-\underline{\lambda}$, then (2) will be violated for all $p>p^{N}$, and hence $p^{C}=p^{N}$. Finally, (2) holds for $p=p^{M}$ if and only if $\delta \geq 1-\bar{\lambda}$, and hence $p^{C}=p^{M}$.

We will treat the maximal cartel price $p^{C}$ in the absence of any antitrust regulation as the benchmark when evaluating the effectiveness of an antitrust regulation in Section 3 and a leniency program in Section 4. Unlike some of the early work, we do not restrict our analysis to the issue whether the monopoly price can be supported by the cartel. Although 
an antitrust regulation may not be effective in preventing the cartel from the monopoly price, we are interested in its relative effectiveness comparing with the case without any antitrust regulation.

In economic applications, one can resort to a simple numerical implementation of our approach: Numerically calculate $\delta(p)=1-\lambda(p)$ and, then, by reversing the dependence, plot the numerical values in the $(\delta, p)$-space for $\delta \in[1-\underline{\lambda}, 1-\bar{\lambda})$. In some cases, such as Example 2 and 3, closed-form solutions for the inverse function $\lambda^{-1}(1-\delta)$ can be derived.

Example 2 Consider a homogeneous Bertrand oligopoly model with linear demand $y=2-p$ and 0 marginal costs. Note that $p^{N}=0$ and $p^{M}=1$. For all $p \in\left(p^{N}, p^{M}\right]$, each of the $n$ firms may deviate by slightly undercutting the others to obtain the full cartel profit, i.e., $\lambda(p)=\frac{1}{n}$ for all $p \in\left(p^{N}, p^{M}\right]$. Consequently, $\underline{\lambda}=\bar{\lambda}=\frac{1}{n}$. Proposition 1 then implies that

$$
p^{C}= \begin{cases}p^{N}, & \text { for } \delta<1-\frac{1}{n}, \\ p^{M}, & \text { for } \delta \geq 1-\frac{1}{n}\end{cases}
$$

Example 3 Consider a (symmetric) heterogenous Bertrand duopoly model. For $i \neq j$, the demand function faced by firm $i$ is given by $y_{i}=1-a p_{i}+b p_{j}$, where $a>b>0$. Firm $i$ 's profit function is

$$
\pi_{i}\left(p_{i}, p_{j}\right)=\left(1-a p_{i}+b p_{j}\right) p_{i}-F
$$

where $F=\frac{a}{(2 a-b)^{2}}$ is the fixed cost that normalizes the model so that $\pi\left(p^{N}\right)=0$ at the static Nash equilibrium price $p^{N}=\frac{1}{2 a-b}$. To find the maximal collusive price,

$$
\max _{p}(1-a p+b p) p \Rightarrow p^{M}=\frac{1}{2(a-b)}>p^{N}
$$

For $p \in\left[p^{N}, p^{M}\right]$, we have $\pi(p)=(1-(a-b) p) p-F$ and $\pi^{o p t}(p)=\frac{(1+b p)^{2}}{4 a}-F$. Recall that $\lambda(p)=\frac{\pi(p)}{\pi^{o p t}(p)}$, we have

$$
\frac{d \lambda(p)}{d p}=\frac{\mathcal{N}(p)}{\pi^{o p t}(p)^{2}} \quad \text { where } \mathcal{N}(p)=\frac{d \pi(p)}{d p} \pi^{o p t}(p)-\pi(p) \frac{d \pi^{o p t}(p)}{d p} .
$$

Observe that

$$
\frac{d \mathcal{N}(p)}{d p}=\frac{d^{2} \pi(p)}{d p^{2}} \pi^{o p t}(p)-\pi(p) \frac{d^{2} \pi^{o p t}(p)}{d p^{2}}=(-2 a+2 b) \pi^{o p t}(p)-\frac{2 b^{2}}{4 a} \pi(p)<0,
$$


which implies that $\mathcal{N}(p)$ is strictly decreasing in $p \in\left[p^{N}, p^{M}\right]$. Since $\mathcal{N}\left(p^{N}\right)=0$ from $\pi\left(p^{N}\right)=\pi^{o p t}\left(p^{N}\right)=0$, we have $\mathcal{N}(p)<0$ for all $p \in\left(p^{N}, p^{M}\right]$ and $\mathcal{N}\left(p^{N}\right)=0$. Therefore, $\lambda(p)$ is strictly decreasing in $p \in\left(p^{N}, p^{M}\right]$. By L'Hôpital's rule, it is easy to check that $\underline{\lambda}=1$, which means that $\lambda(p)$ is continuous for all $p \in\left[p^{N}, p^{M}\right]$. Proposition 1 then implies that if $\delta \geq 1-\bar{\lambda}$, we have $p^{C}=p^{M}$, and if $\delta<1-\bar{\lambda}, p^{C}$ is the solution to $\lambda(p)=1-\delta$, i.e.

$$
-\left(4 a(a-b)+b^{2}(1-\delta)\right) p^{2}+(4 a-2 b(1-\delta)) p-(4 \delta a F+1-\delta)=0 .
$$

Since $\underline{\lambda}=1$ and $\lambda(p)$ is strictly decreasing in $p$, we have that $p^{C}>p^{N}$ for all $\delta>0$ and $p^{C}$ is strictly increasing in $\delta \in(0,1-\bar{\lambda})$. More specifically, we have

$$
\begin{aligned}
p^{C} & = \begin{cases}\frac{2 a-b(1-\delta)+2 \sqrt{a \delta\left(a(1-4 F(a-b))-F b^{2}(1-\delta)\right)}}{4 a(a-b)+b^{2}(1-\delta)}, & \text { for } \delta \in(0,1-\bar{\lambda}), \\
p^{M}=\frac{1}{2(a-b)}, & \text { for } \delta \in[1-\bar{\lambda}, 1),\end{cases} \\
\text { where } \bar{\lambda} & =\frac{4(a-b) a-16(a-b)^{2} a F}{(2 a-b)^{2}-16(a-b)^{2} a F} .
\end{aligned}
$$

The following figure illustrates $p^{C}$ as a function of $\delta \in(0,1)$ for $a=2$ and $b=1$.

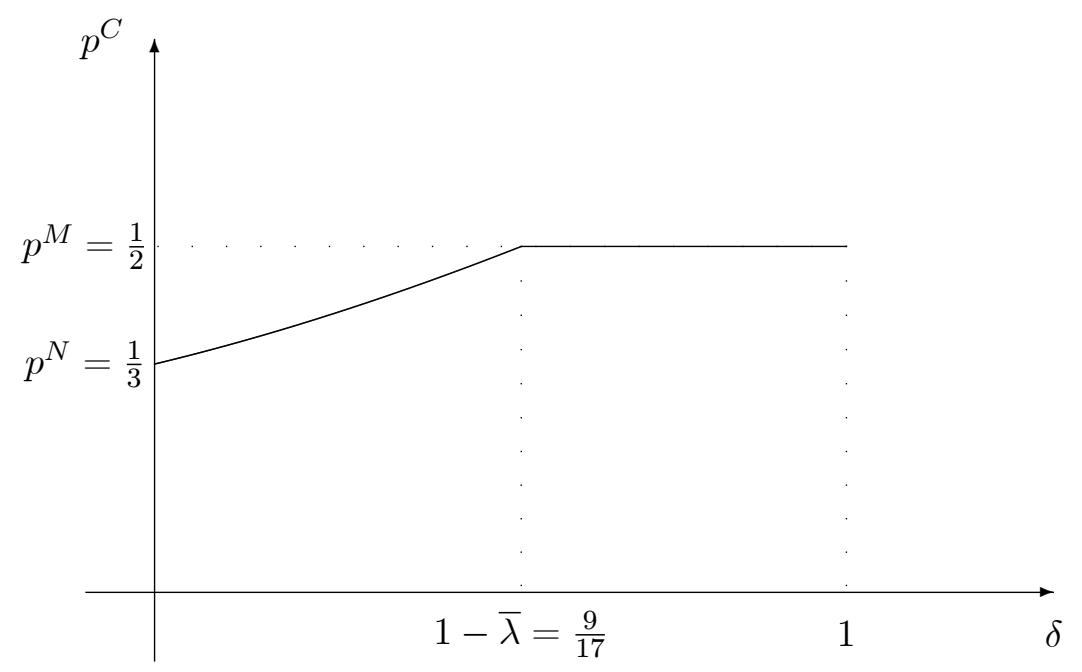

\section{Antitrust Enforcement}

In this section, we examine the impact of traditional antitrust policy. The AA investigates the market outcome in every period with certain probability and implements an antitrust 
policy. Upon being caught, violators will be fined. Given $p \in\left[p^{N}, p^{M}\right]$, the AA will find the firms guilty of collusion with probability $\beta(p) \in[0,1)$. We assume that $\beta\left(p^{N}\right)=0$, and $\beta(p)$ is a non-decreasing differentiable function. As in Rey (2003), hard evidence disappears after one period meaning that if the firms are found guilty of sustaining cartel price $p \in\left(p^{N}, p^{M}\right]$, every firm will have to pay a one-time fine $k(p) \pi(p)$, where $k(p)$ is a non-decreasing continuous function of $p$, such that $k\left(p^{N}\right)=0$ and $k(p)>0$ for all $p \in\left(p^{N}, p^{M}\right] .{ }^{7}$ The function $\beta(\cdot)$ reflects that a higher cartel price might invoke more attention from the AA about cartel abuse and make detection more likely. Any cartel will take the negative effects of its cartel price into account when deciding upon the price. ${ }^{8} 9$

We model the AA as a passive player in this model, while firms are the active players. The detection probability $\beta(\cdot)$ is limited by the resources of the authority, and the fine schedule $k(\cdot)$ is limited by legislation. ${ }^{10}$ Empirical evidence from the OECD countries on antitrust policies, see OECD (2002), reports detection probabilities in the range of one out of seven or six and levels of fines in the range of two to three times the illegal gains from cartel. ${ }^{11}$ This can be formalized as $\frac{1}{7} \leq \beta(p) \leq \frac{1}{6}$ and $2 \leq k(p) \leq 3$. These facts imply

\footnotetext{
${ }^{7}$ Technically speaking, we exclude fixed fines $F>0$, because $k(p) \pi(p)+F$ for all $p \in\left(p^{N}, p^{M}\right.$ ] would imply a discontinuity at $p=p^{N}$. However, for every $F>0$, there exists a continuous approximation that can be made arbitrarily to the discontinuous function and that also satisfies our assumptions. Formally, for every $F>0$ and $\varepsilon>0$, there exists a continuous approximation $k_{\varepsilon}(p)$ such that $k_{\varepsilon}(p) \pi(p)=k(p) \pi(p)+F$ for all $p \in\left(p^{N}+\varepsilon, p^{M}\right]$ and $k_{\varepsilon}\left(p^{N}\right) \pi\left(p^{N}\right)=0$.

${ }^{8}$ The competition stage in Motta and Polo (2003) results if we assume constant functions $\beta(p)=\bar{\beta} \in[0,1]$, $k(p) \pi(p)=\bar{k}>0$ for all $p \in\left[p^{N}, p^{M}\right]$ and restrict the set of feasible prices $p \in\left[p^{N}, p^{M}\right]$ to the discrete set $p^{N}, p^{M}$ and the optimal deviation against $p^{M}$.

${ }^{9}$ The repeated-game version of the model in Harrington (2004) can be obtained as follows: the potential liability $X_{t}$ at period $t$ is equal to, in our terminology, $0 \cdot X_{t-1}+1 \cdot[k(p) \pi(p)+F]$, where $F>0$ is a fixed fine and $k(p) \pi(p)$ represents each firm's liability of private lawsuits. Our detection probability only depends upon the current price and is independent upon the previous one.

${ }^{10}$ According to the sentencing guidelines in most European countries, fines are bounded by either a fixed monetary amount or approximately $10 \%$ of overall annual turnover of the firm. In the US, however, there is no legal upper bound.

${ }^{11}$ The quote from OECD (2002) says: "It is widely agreed that an effective sanction against a cartel should take into account not only the amount of gain realized by the cartel but also the probability that any given cartel will be detected and prosecuted. Because not all cartels are detected, the financial sanction against one that is detected should exceed the gain actually realized by the cartel. Some experts believe that as few as one in six or seven cartels are detected and prosecuted, implying a multiple of at least six. A multiple of three is more commonly cited, however." In the Annex B of OECD (2002), a range of fines between two and three times the illegal profits is reported. The report also mentions countries with a fixed fine independent of the illegal gains. Both systems are used in OECD countries at the moment.
} 
an expected penalty roughly between $30 \%$ to $50 \%$ of illegal gains or monopoly profits, or $\frac{2}{7} \leq \beta(p) k(p) \leq \frac{1}{2}$. Therefore, the AA may not be able to deter these violations. In this paper, we simply assume that $0 \leq \beta(p) k(p)<1$ for all $p \in\left(p^{N}, p^{M}\right]$. This less restrictive assumption implies that the expected fine at any price above the competitive price is lower than the (per period) cartel profit and, therefore, any cartel is tempted to set its price above the competitive price.

An important aspect in assessing the effectiveness of the antitrust regulation is how the cartel members react to detection. In some cases, being caught once is sufficient to deter the cartel members from continuing any such illegal activity in the future. In other cases, the economic sector is notorious for its persistent cartel activities despite many convictions. Persistence means members pay the fines and continue (illegal) business as usual. Since antitrust regulation is involved with all sectors in the economy, the cartel culture within each sector plays an important role in evaluating the effectiveness of the antitrust policy. To allow for a wide range of cartel behavior or to accommodate these differences across sectors, we introduce a cartel culture parameter. Let $\gamma \in[0,1]$ be the probability that the firms will behave competitively after each time the cartel is detected. To put it differently, the cartel culture parameter is interpreted as the probability that the cartel stops its activities after each detection. A high degree of persistence with renewed cartel activity for almost sure is associated with a value of $\gamma$ close to 0 . Similar, a value of $\gamma=1$ means the sector would become competitive after the cartel being detected. ${ }^{12}$ Motta and Polo (2003) assume detection does not dissolve the cartel, i.e., the cartel culture $\gamma=0$, and Harrington (2004) assumes detection dissolves the cartel, i.e., the cartel culture $\gamma=1$. Our cartel-culture parameter captures both models.

Let $V(p)$ be the present value of a cartel member's expected profit if the cartel sets price $p \in\left[p^{N}, p^{M}\right]$ in every period. This present value for each cartel member consists of the

\footnotetext{
${ }^{12}$ The parameter $\gamma$ can be estimated from time series for a particular industry that specify the date of inspection by the authorities and the outcome in terms of whether the firms were found guilty. The fraction of times a convicted cartel was found guilty again at the next investigation serves as a proxy for $\gamma$.
} 
current illegal gains $\pi(p)$ from the cartel, the expected fine $\beta(p) k(p) \pi(p)$, the expected continuation profit of a renewed cartel after detection $\beta(p)(1-\gamma) \delta V(p)$, and the expected continuation profit of not being detected $(1-\beta(p)) \delta V(p)$;

$$
\begin{aligned}
V(p) & =\pi(p)+\beta(p)[-k(p) \pi(p)+(1-\gamma) \delta V(p)]+[1-\beta(p)] \delta V(p) \\
& =\pi(p)-\beta(p) k(p) \pi(p)+\delta[1-\gamma \beta(p)] V(p) .
\end{aligned}
$$

Solving for $V(p)$ yields that

$$
V(p)=\frac{1-\beta(p) k(p)}{1-\delta[1-\gamma \beta(p)]} \pi(p) .
$$

Note that $V\left(p^{N}\right)=\frac{\pi\left(p^{N}\right)}{1-\delta}=0$ due to $\beta\left(p^{N}\right)=0$. For all $p \in\left(p^{N}, p^{M}\right], V(p)>0$ as $\beta(p) k(p)<1$. Since $\beta(p)>0$ and $k(p)>0$ for all $p \in\left(p^{N}, p^{M}\right]$, we have $V(p)<\frac{\pi(p)}{1-\delta}$. This reflects that the possibility of being detected and fined reduces the expected cartel gains. This implies that an antitrust regulation reduces the expected gains from any cartel compared to the benchmark case. Although $\pi(p)$ is assumed to be monotonically increasing in $p, V(p)$ may not be monotonic in $p$ since the fraction in (3) is non-increasing and $\pi(p)$ is non-decreasing in $p \in\left(p^{N}, p^{M}\right]$. To simplify verification of the first statement, denote $B=1-\gamma \beta(p)$ and $A=\beta(p) k(p)$. Then,

$$
\frac{\partial}{\partial p} \frac{1-\beta(p) k(p)}{1-\delta[1-\gamma \beta(p)]}=\frac{\partial}{\partial p} \frac{1-B}{1-\delta(1-A)}=\frac{B^{\prime}[1-\delta(1-A)]-\delta A^{\prime} B}{[1-\delta(1-A)]^{2}} \leq 0
$$

due to $A<1, A^{\prime} \geq 0$ and $B^{\prime} \leq 0$ for all $p \in\left(p^{N}, p^{M}\right]$. The above inequality is strict if $\beta^{\prime}(p)>0$. This property and the product form of $V(p)$ also destroy concavity, as Example 8 below illustrates. So, maximization of $V(p)$ would also require investigation of second-order conditions.

The cartel would become unprofitable, independent of its cartel culture $\gamma \in[0,1]$, if the AA could set its policy $\beta(p) k(p)>1$, i.e., the expected fine is above $100 \%$ of illegal gains. As we have discussed, the current practice within the OECD countries indicates that $\beta(p) k(p)$ is at most $50 \%$. Inspection of (3) also shows that cartel profit is decreasing with respect to 
its culture parameter $\gamma$ (higher $\gamma$ means less persistence). How this affects antitrust policy will be dealt with later.

Moreover, the cartel has its own destabilizing forces working from within. These destabilizing forces are the incentives of individual cartel members to cheat on the cartel's implicit agreement. In order to express these incentives, it is necessary that we describe what happens within the cartel after some of its members cheat. As motivated in Section 2, cartel members adopt the following modified grim-trigger strategy profile to sustain a cartel price of $p>p^{N}$ :

1. Firms continue to set a price $p>0$ with probability $1-\gamma$ every time the AA detects their fraud (and with probability $\gamma$ behave competitively ever after).

2. Any deviation by some cartel members leads to the perfectly competitive equilibrium $p^{N}$ in every period ever after.

This modified grim-trigger strategy profile assumes that the trust is gone forever when some members cheat.

Given such an implicit cartel agreement, the profit from a unilateral deviation is equal to the short term gain of $\pi^{o p t}(p)$ in the current period, followed the normalized profit from the competitive equilibrium forever. ${ }^{13}$ In terms of Abreu et al. (1986), individual deviations are punished by the symmetric stationary SPE $p^{N}$. Consequently, the necessary and sufficient condition to support a cartel price $p \in\left(p^{N}, p^{M}\right]$ is $V(p) \geq \pi^{\text {opt }}(p)$ :

$$
\frac{1-\beta(p) k(p)}{1-\delta[1-\gamma \beta(p)]} \pi(p) \geq \pi^{o p t}(p)
$$

which implies that

$$
\lambda(p) \geq \Lambda(p) \equiv \frac{1-\delta[1-\gamma \beta(p)]}{1-\beta(p) k(p)},
$$

\footnotetext{
${ }^{13}$ We assume that after a firm deviates, this firm will not be found guilty due to its heterogeneous price set, while the other firms may still be found guilty and fined by the anti-trust authority.
} 
where $\Lambda(p)$ is a continuous function on $\left[p^{N}, p^{M}\right]$. Obviously, $p=p^{N}$ satisfies inequality (5) and it would reduce to (1) if $\beta(p)=0$ for all $p \in\left(p^{N}, p^{M}\right]$. So, we can conclude that (5) generalizes the benchmark model of absence of any regulation discussed in section 2 .

Before continuing the economic analysis, we first establish the following properties of the function $\Lambda(\cdot)$ as defined in (5).

Lemma $4 \Lambda(p)$ is non-decreasing in $p$ and increasing when either $\beta^{\prime}(p)>0$ or $k^{\prime}(p)>0$. Moreover, $\Lambda(p)$ is increasing in $\gamma, k(p)$ and $\beta(p)$ and decreasing in $\delta$.

Proof. Taking derivative of $\Lambda(p)$ with respect to $p$, we have

$$
\frac{d \Lambda(p)}{d p}=\frac{\delta \gamma \beta^{\prime}(p)[1-\beta(p) k(p)]+\left[\beta^{\prime}(p) k(p)+\beta(p) k^{\prime}(p)\right][1-\delta(1-\gamma \beta(p))]}{[1-\beta(p) k(p)]^{2}} \geq 0,
$$

due to our assumptions on $\beta(p)$ and $k(p)$. In addition, when either $\beta^{\prime}(p)>0$ or $k^{\prime}(p)>0$, the above inequality is strict. Next, $\hat{\beta}(p)>\beta(p)$ implies

$$
[(1-\delta) k(p)+\gamma \delta][\hat{\beta}(p)-\beta(p)]>0 \Longleftrightarrow \frac{1-\delta[1-\gamma \hat{\beta}(p)]}{1-\hat{\beta}(p) k(p)}>\frac{1-\delta[1-\gamma \beta(p)]}{1-\beta(p) k(p)} .
$$

The effects of $\gamma, \delta$ and $k(p)$ are obvious.

Lemma 4 implies that the right-hand side of (5) increases when $\gamma$ increases. Hence, a lower $\gamma$ would weaken (5). This implies that the more persistent the collusion is (i.e., lower $\gamma$ ), the easier it is to sustain a cartel price. Similar, the right-hand side of (5) is decreasing in $\delta$, meaning that an increase in $\delta$ would relax (5). As the firms care more about future, it becomes easier to sustain the same cartel price. Also an overall increase in detection probabilities $\beta(p)$ or fines $k(p)$ would make collusion harder to sustain.

The variable of interest is the maximal cartel price. Higher cartel prices put an upward pressure on the right-hand side of (5). This reduces the sustainability of $p$ and only industries with relative high values of $\lambda(p)$ close to 1 might withstand this pressure, meaning the short-term gains of blowing up the cartel must be relatively close to the cartel profit $\pi(p)$. Since the weak monotonicity properties of $\Lambda(p)$ (non-decreasing) and $\lambda(p)$ (nonincreasing) are opposite, the intersection $\lambda(p)=\Lambda(p)$ is either a unique price or a closed 
subinterval of $\left[p^{N}, p^{M}\right]$. Furthermore, if $p \in\left(p^{N}, p^{M}\right]$ is any such price, then all lower prices can also be sustained by the cartel. So, the range of prices that can be sustained as cartel prices in (5) is a closed subinterval of $\left[p^{N}, p^{M}\right]$ and the maximal cartel price is bounded by $\max \{p: \lambda(p)=\Lambda(p)\}$.

Note that $\Lambda\left(p^{N}\right)=1-\delta$. It is straightforward that $p=p^{N}$ satisfies (5) since $\lambda\left(p^{N}\right)=$ $1>\Lambda\left(p^{N}\right)$. Given the monotonicity of $\lambda(p)$ and $\Lambda(p)$, we have a well-defined maximal price on $\left[p^{N}, p^{M}\right]$ for all $\delta \in[0,1)$ and $\gamma \in[0,1]$. Under the antitrust policy discussed in this section, the maximal cartel price is given by

$$
p^{R}=\max _{p \in\left[p^{N}, p^{M}\right]} p, \quad \text { s.t. }(5) .
$$

where superscript $R$ refers to the presence of antitrust regulation. Program (6) is a welldefined program since $p \in\left[p^{N}, p^{M}\right]$ and (5) induces a closed subinterval of $\left[p^{N}, p^{M}\right]$. Because $V(p)$ may not be monotonic in $p$, the maximal cartel price $p^{R}$ may not maximize the expected profit to each cartel member. Nevertheless, $p^{R}$ is the worst social outcome under such antitrust regulation.

Comparing (2) and (6), our next result shows that antitrust regulation may lower the maximal cartel price in general.

Proposition 5 For all parameter values, we have $p^{N} \leq p^{R} \leq p^{C} \leq p^{M}$.

Proof. By Lemma 4 and $\Lambda\left(p^{N}\right)=1-\delta$, we have $\Lambda(p) \geq 1-\delta$. In other words, any $p$ that satisfies (5) must also satisfies (1), which concludes the proof.

To be more specific, similar to Proposition 1, we now derive the thresholds on the discount factor $\delta$ for different values of $p^{R}$. Note that (5) can be rewritten as

$$
\delta \geq \Delta(p) \equiv \frac{1-\lambda(p)[1-\beta(p) k(p)]}{[1-\gamma \beta(p)]} .
$$

Lemma $6 \Delta(p)$ is continuous and non-decreasing in $p \in\left(p^{N}, p^{M}\right], \Delta(p) \geq 1-\lambda(p)$ (with strict inequality when $\beta(p)>0)$ and increasing in $k(p)$ and $\beta(p)$. 
Proof. Since $\lambda(p), \beta(p)$, and $k(p)$ are all continuous in $p \in\left(p^{N}, p^{M}\right]$, so is $\Delta(p)$. Taking derivative of $\Delta(p)$ with respect to $p$, we have

$$
\frac{d \Delta(p)}{d p}=\frac{-\lambda^{\prime}(p)[1-\beta(p) k(p)]+\lambda(p)\left[\beta^{\prime}(p) k(p)+\beta(p) k^{\prime}(p)\right]}{[1-\gamma \beta(p)]}+\Delta(p) \frac{\gamma \beta^{\prime}(p)}{1-\gamma \beta(p)} \geq 0
$$

due to $\lambda^{\prime}(p) \leq 0, \beta^{\prime}(p) \geq 0$ and $k^{\prime}(p) \geq 0$. Note also that

$$
\Delta(p) \equiv \frac{1-\lambda(p)[1-\beta(p) k(p)]}{[1-\gamma \beta(p)]} \geq 1-\lambda(p)[1-\beta(p) k(p)] \geq 1-\lambda(p) .
$$

The last inequality is strict when $\beta(p)>0$. Next, $\hat{\beta}(p)>\beta(p)$ implies

$[\gamma(1-\lambda(p))+\lambda(p)][\hat{\beta}(p)-\beta(p)]>0 \Longleftrightarrow \frac{1-\lambda(p)[1-\hat{\beta}(p) k(p)]}{1-\gamma \hat{\beta}(p)}>\frac{1-\lambda(p)[1-\beta(p) k(p)]}{1-\gamma \beta(p)}$

The effect of $k(p)$ is obvious.

Proposition 7 Under antitrust regulation, the maximal cartel price $p^{R}$ is non-decreasing in $\delta \in(0,1)$ and non-increasing in $\gamma \in[0,1]$. Furthermore, we have

$$
\begin{array}{ll}
p^{R}=p^{N}, & \text { for } \delta \in(0,1-\lambda), \\
p^{R} \in\left[p^{N}, p^{M}\right), & \text { for } \delta \in\left[1-\lambda, \Delta\left(p^{M}\right)\right), \\
p^{R}=p^{M}, & \text { for } \delta \in\left[\Delta\left(p^{M}\right), 1\right),
\end{array}
$$

An overall increase in detection $\beta(p)$ or $k(p)$ shifts $\Delta\left(p^{M}\right)$ and the entire curve to the right.

Proof. Lemma 4 and monotonicity of $\lambda(p)$ imply that $p^{R}$ is non-decreasing in $\delta$ and nonincreasing in $\gamma$. Note that $\Delta\left(p^{N}\right)=0$ and $\lim _{\varepsilon \rightarrow 0^{+}} \Delta\left(p^{N}+\varepsilon\right)=1-\underline{\lambda}$. When $\delta \in(0,1-\underline{\lambda})$, (5) fails for all $p \in\left(p^{N}, p^{M}\right]$ and, hence, $p^{R}=p^{N}$. For $\Delta\left(p^{M}\right) \leq \delta<1$, (5) holds at $p=p^{M}$ and, hence, $p^{R}=p^{M}$. Otherwise, we have $p^{R} \in\left[p^{N}, p^{M}\right)$.

Clearly, inequality (7) is more restrictive than (1) implying that introduction of antitrust regulation with positive probability of being caught restricts the set of discount factors for which collusion can be sustained for every possible price $p \in\left(p^{N}, p^{M}\right]$. This implies that cartel stability is reduced compared to benchmark case. Moreover, the fact that $\Delta(p)$ is non-decreasing in $p$ implies that the antitrust policy discussed in this section might be more effective for more grave violations (i.e. collusion on higher prices). 
Proposition 7 also implies that

$$
0<1-\underline{\lambda} \leq 1-\bar{\lambda} \leq \Delta\left(p^{M}\right)<1
$$

Depending on the discount factor $\delta$, Propositions 1,7 , and 5 imply the following results:

$$
\begin{array}{ll}
p^{N}=p^{R}=p^{C}=p^{N}, & \text { if } 0<\delta<1-\underline{\lambda}, \\
p^{N}<p^{R}<p^{C}<p^{M}, & \text { if } 1-\underline{\lambda} \leq \delta<1-\bar{\lambda}, \\
p^{N}<p^{R}<p^{C}=p^{M}, & \text { if } 1-\bar{\lambda} \leq \delta<\Delta\left(p^{M}\right), \\
p^{M}=p^{R}=p^{C}=p^{M}, & \text { if } \Delta\left(p^{M}\right) \leq \delta<1 .
\end{array}
$$

When $\Delta\left(p^{M}\right) \leq \delta<1$, the antitrust policy is not effective to deter the cartel from setting its monopoly price. In particular, antitrust enforcement would not be able to reduce the cartel price below the monopoly level $\left(p^{R}=p^{M}\right)$, when interval $\delta \in\left[\Delta\left(p^{M}\right), 1\right)$ is non-empty. This implies that necessary and sufficient condition for $p^{R}=p^{M}$ is $\Delta\left(p^{M}\right)<1$. By (7), $\Delta\left(p^{M}\right)<1$ requires that condition (5) for cartel stability is broken at $\delta=1$, i.e.,

$$
\frac{\lambda\left(p^{M}\right)}{\gamma}>\frac{\beta\left(p^{M}\right)}{1-\beta\left(p^{M}\right) k\left(p^{M}\right)} .
$$

This inequality can be numerically verified in applications, it is written to separate the industry characteristics $(\gamma, \lambda(p))$ from the policy instruments $(\beta(p), k(p))$.

Given a particular antitrust policy, it is interesting to investigate whether this policy can eradicate the monopoly price for all cartel cultures. Solving condition (8) for $\gamma$ yields

$$
\gamma<\frac{\lambda\left(p^{M}\right)\left[1-k\left(p^{M}\right) \beta\left(p^{M}\right)\right]}{\beta\left(p^{M}\right)}
$$

To destabilize cartels for all possible cartel cultures $\gamma \in[0,1]$, the right-hand side must be negative, i.e., $\beta\left(p^{M}\right) k\left(p^{M}\right)>1$. Hence, under any cartel policy that satisfies condition $0<\beta(p) k(p)<1$ for all $p \in\left(p^{N}, p^{M}\right]$, industries that are notorious for persistent cartel behavior ( $\gamma$ close to 0$)$ cannot be eradicated by the antitrust policy unless one is willing to adopt a policy that fully takes away the illegal gains (i.e. $k(p) \beta(p)>1$ for all $p \in\left(p^{N}, p^{M}\right]$ ).

Another important conclusion is related to the impact of parameter $\lambda$ on sustainability of the monopoly price. Recall, that $\lambda(\cdot)$ can be viewed as a ratio of individual illegal gains from cartel formation to profits from unilateral optimal deviation against the cartel. This implies 
that $\lambda(\cdot)$ also relates to particular characteristics of a sector, such as incentives to deviate or degree of cartel stability in the sector. Hence, conditions of the above proposition imply that in sectors were the degree of cartel stability is higher $(\lambda(\cdot)$ closer to 1$)$, the likelihood of sustaining society's worst price $p^{M}$ also increases, since $\partial \Delta / \partial \lambda<0$. So that in these sectors, we should expect that antitrust policy will be less effective compared to the sectors with lower $\lambda(\cdot)$.

The main message of analysis in this section is a mixed blessing for antitrust regulation. On the one hand, proposition 7 identifies non-empty sets of parameter values for which antitrust regulation is effective in reducing the maximal price the cartel can sustain, i.e., $p^{R}<p^{C}$. On the other hand, as long as the authority or the legal system obeys condition (8), there always will remain a large non-empty set of parameter values for which $p^{R}=p^{M}$, meaning the antitrust policy is totally ineffective on this set.

The following example illustrates the issues discussed in this section.

Example 8 Reconsider the homogeneous Bertrand oligopoly of Example 2. Suppose that $\beta(p)=\beta p$ and $k(p)=k$, where $k \beta<1$. Then, (6) becomes

$$
p(\delta, \gamma)=\max _{p \in[0,1]} p, \quad \text { s.t. } \frac{1}{n} \geq \frac{1-\delta+\gamma \delta \beta p}{1-k \beta p} .
$$

Note that $p=0$ is feasible in the quadratic constraint if and only if $\delta \geq 1-\frac{1}{n}$. The constraint can be rewritten as

$$
p \leq \frac{1-n(1-\delta)}{(n \gamma \delta+k) \beta}
$$

which is the solution to the problem whenever it is between 0 and 1. The right hand side is increasing in $\delta$. To summarize, we have

$$
p^{R}= \begin{cases}0, & \text { if } \delta<1-\frac{1}{n} \\ \min \left\{1, \frac{1-n(1-\delta)}{(n \gamma \delta+k) \beta}\right\}, & \text { if } 1-\frac{1}{n} \leq \delta<1\end{cases}
$$

Depending on the values of the parameters, $p^{R}$ may not be equal to $p^{M}=1$ for all $\delta \in$ $(0,1)$, such as if $(n \gamma+k) \beta>1$. Since $\beta k<1$, this condition can hold only when $n \gamma$ is 
sufficiently large. For sectors with a small number of firms and a cartel culture parameter $\gamma$ that is sufficiently close to 0 , the monopoly price will not be eradicated by the antitrust regulation in this example. We can also see how each parameter value affects the maximal cartel price under the antitrust policy. Both possible cases for the curve of maximal prices $p^{R}$ are illustrated by the following figure, where the vertical dotted line at $\delta=1-\frac{1}{n}$ represents the discontinuous jump in $p^{N}=0$ to $p^{M}=1$ under the benchmark case in Example 2.
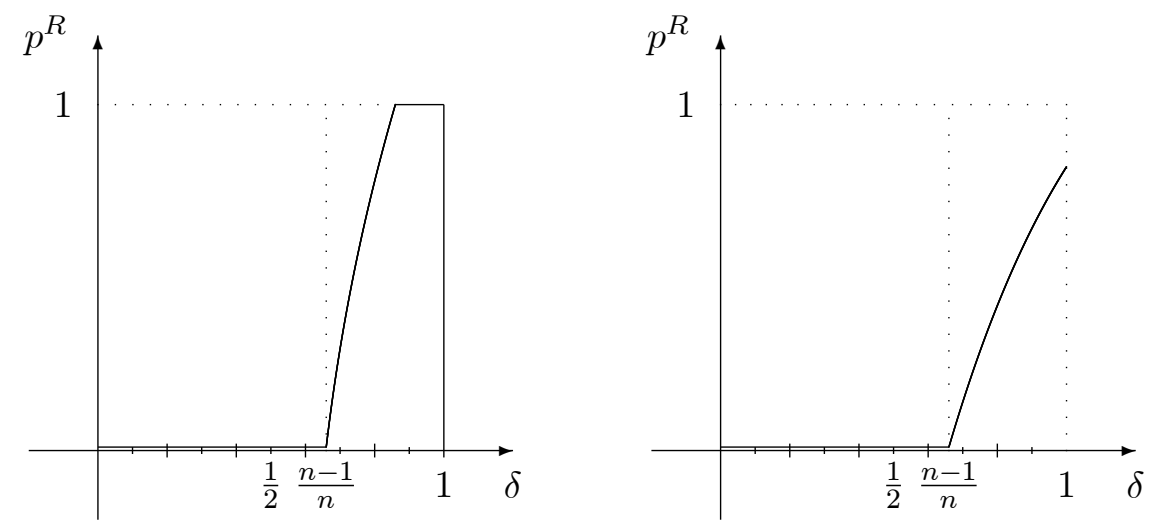

We conclude this example, by relating our results to Harrington (2004 and 2005). By (3), $V(p)=\frac{1-\beta k p}{1-\delta(1-\gamma \beta p)} \cdot \frac{(2-p) p}{n}$ fails both monotonicity and concavity on $\left[p^{N}, p^{M}\right] \cdot{ }^{14}$ The function is single peaked. For parameter values $k=3, \beta=\frac{1}{6}$ and $\gamma=\frac{2}{3}$, neglecting the equilibrium conditions as in Harrington (2005), MAPLE obtains from $\partial V(p) / \partial p=0$ the profit-maximizing cartel price

$$
\frac{-27(1-\delta)+3 \sqrt{65 \delta^{2}-146 \delta+81}}{4 \delta} \in\left[0, \frac{3}{4}\right)
$$

for all $\delta \in[0,1]$. The profit-maximizing cartel price is decreasing on $[0,1]$. Introducing the equilibrium conditions, implies that the profit-maximizing cartel price is the minimum of the above expression and $p^{R}$ (the figure to the left in the last two figures applies). These two price curves intersect at $\delta \approx 955$. So, on the interval $[0,0.955]$, the profit-maximizing cartel price coincides with $p^{R}$, while for the interval $(.955,1]$ the profit-maximizing cartel price is lower

\footnotetext{
${ }^{14}$ Without going into details, for $\delta$ close to 1 it holds that $\partial V(p) / \partial p>0$ for $p$ close to 0 , while $\partial V(p) / \partial p<$ 0 for $p$ close to 1 . For parameter values $k=3, \beta=\frac{1}{6}, \gamma=\frac{2}{3}$ and $\delta=.99$, software package MAPLE returns the inflexion point $p=0.64433 \in\left[p^{N}, p^{M}\right]$ when solving $\partial^{2} V(p) / \partial p^{2}=0$.
} 
than $p^{R}$ because the equilibrium conditions are non-binding. This result confirms the assertion in Harrington (2004 and 2005) that the equilibrium conditions are always non-binding for sufficiently large $\delta<1$. Our results, however, also show that the profit-maximizing cartel price is non-monotone on $[0,1]$. Moreover, as $\delta$ goes to 1 , Harrington (2004 and 2005) predict a limit cartel price that is equal to the competitive price $p^{N}$. This implies that for $\delta$ close to 1 , the profit-maximizing cartel price seriously underestimates the potential maximal damage to society (consumers) under sustainable cartel behavior. The following figure illustrates these findings, where the solid curve is the lower envelope of the two dotted curves that represent the discussed price curves.

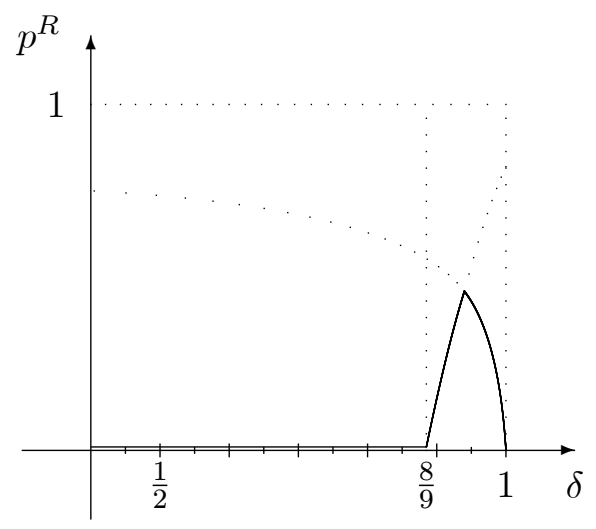

\section{Leniency Programs}

As we have shown, traditional antitrust policy alone may not be effective to eradicate all cartel prices. One revision of antitrust policy that is considered recently in most of the EU countries and in the US is what is often referred to as a leniency program. ${ }^{15}$ The essence of such program is that cartel members can self-report their misbehavior to the AA and that in return self-reporting firms have to pay less severe penalties. According to the current leniency programs in Europe, a self-reporting firm will receive a reduced fine that depends on how many other firms have self reported already. The US can be seen as an extreme variant of the EC system in which the first self-reporting firm is fully exempted and all

\footnotetext{
${ }^{15}$ See OECD (2002), (EC 2002), and (D.O.J. 1993) for detailed information about structure of leniency programs in most EU countries and in the US.
} 
subsequent self-reporting firms will have to pay the full fine. In this section, we examine antitrust policies with ex-ante leniency programs and study its impact on the maximal cartel price. We will characterize the necessary and sufficient conditions under which introducing leniency programs as part of antitrust policy is effective. This implies that ex-ante leniency programs are not by its mere presence effective.

We incorporate a simplified ex-ante leniency program into the model as follows. In every period, firms first choose their cartel price simultaneously as before. Upon a cartel being formed, i.e., all choose $p \in\left(p^{N}, p^{M}\right]$ and cartel profits are realized, firms then independently and simultaneously choose whether to self-report to the AA before any investigation begins. If at least one firm self-reports, all cartel members are convicted and fined. If no firm selfreports, the authority investigates the industry with probability $\beta(\cdot)$ as in Section 3. The stage game in any period is a two-stage game, hence the repeated game model is an infinitely repeated sequential game, see e.g. Wen (2002). As in Rey (2003), we assume the hard case of public self-reporting meaning that if someone reveals information, this cannot be kept secret and all other cartel members immediately know this.

There are two situations that are particularly important to our later analysis. If none of the firms self-reports, then the expected profit to a firm during a period is simply equal to $\pi(p)-\beta(p) k(p) \pi(p)$. However, if a firm self-reports while none of the other firms selfreports, the profit to the (only) self-reporting firm is $\pi(p)-\alpha(p) \pi(p)$ in the period, where $\alpha(p) \pi(p)$ is the reduced fine that the first self-reporting firm has to pay. Assume that $\alpha\left(p^{N}\right)=0, \alpha(p) \leq k(p)$ and $\alpha(p)$ is non-increasing and continuous for all $p \in\left(p^{N}, p^{M}\right]$ with $\lim _{p \downarrow p^{N}} \alpha(p)=\underline{\alpha}$. Note that it is possible that $\alpha(p)<0$, meaning a self-reporting firm will be rewarded. The absence of a leniency program can be treated as a special case by setting $\alpha(p)=k(p)$, meaning self-reporting does not yield any reduction of the fine for cooperating with the authorities. The US leniency program is captured by $\alpha(p)=0$ and the EC leniency program corresponds to $0 \leq \alpha(p) \leq k(p)$, where both $\alpha(p)$ and $k(p)$ are constant and $\alpha(p)$ 
can be reinterpreted as a constant percentage of fine reduction. ${ }^{16} 17$

The issue of multiple self-reporting firms has been much discussed in the literature, see e.g. Spagnolo (2007) and Motchenkova (2004), because the largest fine reduction in many countries applies only to the first self-reporting firm and all other firms would pay higher fines up to the full fine. Given that the decisions to self-report are taken simultaneously, our model cannot capture the "first" self-reporting firm. Since we study the subgame perfect equilibrium in which no firm will self-report along the equilibrium outcome path, it is not necessary to specify the expected profits in case more than a single firm unilaterally deviates by self-reporting. So, multiple self-reporting firms are not a concern in our analysis of the symmetric subgame perfect equilibrium in which no firm will self-report along the equilibrium outcome path, since only unilateral decision matters. ${ }^{18}$

Since the objective of a leniency program is to provide an incentive for the individual firm to self-report, such policy adds a second destabilizing force to the cartel. As in the previous sections, we characterize the maximal cartel price sustainable by the following modified grim-trigger strategy profile:

1. Firms continue to set a price $p>p^{N}$ with probability $1-\gamma$ every time after the AA detects their fraud (and with probability $\gamma$ behave competitively thereafter).

2. Any deviation by one of the cartel members (either setting a lower price or selfreporting) leads to the perfectly competitive equilibrium in every period thereafter.

As before, this modified grim-trigger strategy profile assumes that the trust is gone forever when a cartel member either undercuts the cartel price or self-reports. Given the implicit cartel agreement, the expected profits of sustaining the cartel price $p$ are equal to $V(p)$ in $(3)$

\footnotetext{
${ }^{16}$ The competition stage with ex-ante leniency in Motta and Polo (2003) results if we assume the constant function $\alpha(p)=\bar{\alpha} \in[0, \bar{k}]$, where $k(p)=\bar{k}$. Spagnolo (2004) and Rey (2003) correspond to $\bar{\alpha} \in(-\infty, \bar{k}]$.

${ }^{17}$ In our terminology, Harrington and Chen (2006) assumes $\alpha(p) \pi(p)=k(p) \pi(p)+\theta F$, where $F>0$ is a fixed fine, $\theta \in[0,1]$ and $k(p) \pi(p)$ represents each firm's liability of private lawsuits. Note that $\underline{\alpha}=\theta F$.

${ }^{18}$ Although the percentage of fine reductions specified by the current leniency programs in the US and EU do not depend on the severity of the offense, our model allows fines and percentages of fine reductions to vary with the severity of collusion. Our model also allows for rewarding self-reporting firms, although it has not been implemented in practice.
} 
for the same reason. In this modified grim-trigger strategies, there are two types of unilateral one-stage deviations: undercut the cartel price and self-report to the AA. Each type of such deviation can be analyzed independently from the other. Inequality (5) is still the necessary and sufficient condition under which no cartel member would undercut the cartel price.

The expected continuation profit of the one-stage deviation by self-reporting consists of paying the reduced fine $\alpha(p) \pi(p)$ in the current period followed by the continuation profits from the competitive equilibrium $p^{N}$ forever after due to the breakdown of trust. Therefore, a firm will not self-report if and only if

$$
-\alpha(p) \pi(p) \leq \beta(p)[-k(p) \pi(p)+\delta(1-\gamma) V(p)]+[1-\beta(p)] \delta V(p),
$$

which simplifies to

$$
1-\alpha(p) \leq \frac{V(p)}{\pi(p)}=\frac{1}{\Lambda(p)} .
$$

Condition (9) implies that, in order to break the silence of no self-reporting, the AA should choose a fine reduction $\alpha(p) \leq k(p)$ that is sufficiently low. More specifically, the leniency program fails to be effective if $\alpha(p) \geq 1$, i.e., the reduced fine should never be more than the firm's profit in a single period. When $\Lambda(p)<1$, which is relevant for sufficiently large $\delta$ and sufficiently low $\gamma$, the AA would have to compensate the self-reporting firm for voluntarily giving up its future expected rents from staying in the cartel, i.e., $\alpha(p)$ must be negative. ${ }^{19}$ Hence, from here on we only consider the case of $\alpha(p)<1$ and investigate whether the leniency program is effective. Accordingly, (9) can be rewritten as

$$
\frac{1}{1-\alpha(p)} \geq \Lambda(p)
$$

Under a leniency program, the maximal cartel price is given by

$$
p^{L}=\max _{p \in\left[p^{N}, p^{M}\right]} p, \quad \text { s.t. (5) and (10), }
$$

\footnotetext{
${ }^{19}$ Possibility and effectiveness of rewards has also been extensively studied in Spagnolo (2004) or Spagnolo (2007).
} 
where superscript $L$ refers to the presence of the leniency program. In Section 3, we have shown that (5) and $p \in\left[p^{N}, p^{M}\right]$ imply that $p \in\left[p^{N}, p^{R}\right]$. Now define

$$
\tilde{p} \equiv \max _{p \in\left[p^{N}, p^{M}\right]} p, \quad \text { s.t. }(10)
$$

First, notice that the competitive price $p^{N}$ satisfies (10). Since $\Lambda(p)$ and $\alpha(p)$ are both continuous for $p \in\left(p^{N}, p^{M}\right],(10)$ also induces a non-empty closed subset of $\left[p^{N}, p^{M}\right]$. More specifically, if (10) fails for all $p \in\left(p^{N}, p^{M}\right]$, i.e.,

$$
\frac{1}{1-\lim _{p \downarrow p^{N}} \alpha(p)}<\Lambda\left(p^{N}\right),
$$

then $\tilde{p}=p^{N}$. If $(10)$ holds at $p^{M}$, then $\tilde{p}=p^{M}$. Otherwise, since $\alpha(\cdot)$ is non-increasing by assumption and $\Lambda(\cdot)$ is non-decreasing by Lemma 4 , we have a unique $\tilde{p} \in\left(p^{N}, p^{M}\right)$ such that (10) holds with equality at $\tilde{p}$.

The analysis thus far implies the following result:

Proposition 9 Under antitrust regulation with a leniency program, we have

$$
p^{L}=\min \left\{p^{R}, \tilde{p}\right\} \in\left[p^{N}, p^{R}\right]
$$

We illustrate this result for the case $p^{L}=\tilde{p}<p^{R}$ under constant $\lambda(\cdot)$ and $\alpha(\cdot)$ functions by the following figure.

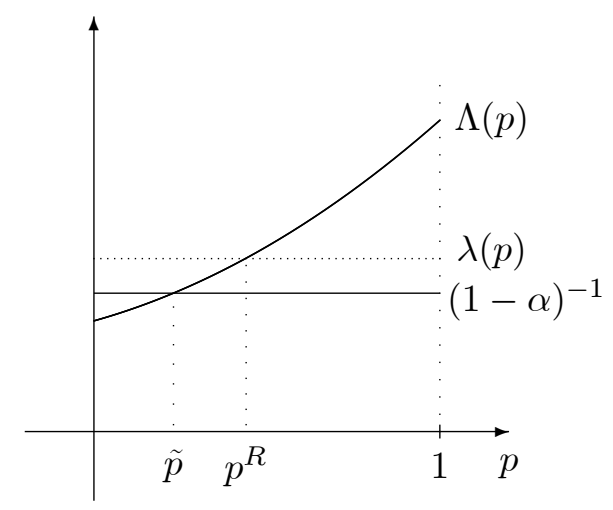

Recall that (4) is equivalent to $\delta \geq \Delta(p)$. Similarly, constraint (9) is equivalent to

$$
\delta \geq \Gamma(p) \equiv \frac{[\beta(p) k(p)-\alpha(p)]}{[1-\gamma \beta(p)][1-\alpha(p)]} .
$$


Note that $\Gamma\left(p^{N}\right)=0$ and $\Gamma(p)$ is a non-decreasing and continuous function of $p \in\left(p^{N}, p^{M}\right]$. The following result characterizes the maximal cartel price as a function of the discount factor $\delta$.

Proposition 10 The maximal cartel price $p^{L}$ is non-decreasing in $\delta \in(0,1)$ and nonincreasing in $\gamma \in(0,1)$. Furthermore, we have

$$
\begin{array}{ll}
p^{L}=p^{N}, & \text { for } \delta \in\left(0, \max \left\{\Delta\left(p^{N}\right), \Gamma\left(p^{N}\right)\right\}\right), \\
p^{L} \in\left[p^{N}, p^{M}\right), & \text { for } \delta \in\left[\max \left\{\Delta\left(p^{N}\right), \Gamma\left(p^{N}\right)\right\}, \max \left\{\Delta\left(p^{M}\right), \Gamma\left(p^{M}\right)\right\}\right), \\
p^{L}=p^{M}, & \text { for } \delta \in\left[\max \left\{\Delta\left(p^{M}\right), \Gamma\left(p^{M}\right)\right\}, 1\right) .
\end{array}
$$

Proof. Observe that $\Lambda(\cdot)$ is decreasing in $\delta \in(0,1)$ and increasing in $\gamma \in(0,1)$. Increasing $\delta$ and/or decreasing $\gamma$ will relax both (4) and (10). Therefore, $p^{L}$ is non-decreasing in $\delta \in(0,1)$ and non-increasing in $\gamma \in(0,1)$.

For $\delta \in\left(0, \max \left\{\Delta\left(p^{N}\right), \Gamma\left(p^{N}\right)\right\}\right)$, either (4) or (10) fails for all $p \in\left(p^{N}, p^{M}\right]$. Therefore, we have $p^{L}=p^{N}$. For $\delta \in\left[\max \left\{\Delta\left(p^{M}\right), \Gamma\left(p^{M}\right)\right\}, 1\right)$, both (4) and (10) hold at $p^{M}$, and hence $p^{M}$ is the maximal cartel price. Otherwise, for $\delta \in\left[\max \left\{\Delta\left(p^{N}\right), \Gamma\left(p^{N}\right)\right\}, \max \left\{\Delta\left(p^{M}\right), \Gamma\left(p^{M}\right)\right\}\right)$, it must be the case that $p^{L} \in\left[p^{N}, p^{M}\right)$.

Combining Proposition 7 and 9, we obtain

$$
\begin{array}{ll}
p^{N}=p^{L}=p^{R}=p^{N}, & \text { for } \delta \in\left(0, \min \left\{\Delta\left(p^{N}\right), \Gamma\left(p^{N}\right)\right\}\right), \\
p^{N}=p^{L} \leq p^{R}<p^{M}, & \text { for } \delta \in\left[\min \left\{\Delta\left(p^{N}\right), \Gamma\left(p^{N}\right)\right\}, \max \left\{\Delta\left(p^{N}\right), \Gamma\left(p^{N}\right)\right\}\right], \\
p^{N}<p^{L}<p^{R}<p^{M}, & \text { for } \delta \in\left(\min \left\{\Delta\left(p^{N}\right), \Gamma\left(p^{N}\right)\right\}, \min \left\{\Delta\left(p^{M}\right), \Gamma\left(p^{M}\right)\right\}\right), \\
p^{N}<p^{L}<p^{R}=p^{M}, & \text { for } \delta \in\left(\min \left\{\Delta\left(p^{M}\right), \Gamma\left(p^{M}\right)\right\}, \max \left\{\Delta\left(p^{N}\right), \Gamma\left(p^{N}\right)\right\}\right), \\
p^{M}=p^{L}=p^{R}=p^{M}, & \text { for } \delta \in\left[\max \left\{\Delta\left(p^{N}\right), \Gamma\left(p^{N}\right)\right\}, 1\right) .
\end{array}
$$

The policy relevant question is then whether such a leniency program is effective to upset the cartel price $p \in\left(p^{N}, p^{M}\right]$. Suppose that without the leniency program, it is possible to sustain a cartel price $p \in\left(p^{N}, p^{R}\right]$ meaning $\Lambda(p) \leq \lambda(p)$ holds. The leniency program is effective to upset the cartel price $p$ if and only if (10) fails at $p$. Accordingly,

$$
\frac{1}{1-\alpha(p)}<\Lambda(p) \leq \lambda(p) \Longleftrightarrow \alpha(p)<1-\frac{1}{\Lambda(p)} \leq 1-\frac{1}{\lambda(p)} \leq 0 .
$$

Before discussing (13), we first state the following result. 
Lemma 11 The upper bound $1-\frac{1}{\Lambda(p)}$ is non-decreasing in $p$ and increasing in $p$ if either $\beta^{\prime}(p)>0$ or $k^{\prime}(p)>0$. Moreover, $1-\frac{1}{\Lambda(p)}$ is increasing in $\gamma, k(p)$ and $\beta(p)$ and decreasing in $\delta$.

Proof. All results follow immediately from Lemma 4 after observing that $1-\frac{1}{\Lambda}$ responds similar to changes in variables and parameters as $\Lambda$.

The upper bound (13) on $\alpha(p)$ has several interesting and important implications. First, it implies that in order for the ex-ante leniency program to be effective, the first self-reporting firm needs to be rewarded as $\alpha(p)<0$. This result is quite intuitive. Since a firm's future expected cartel profit is positive (i.e. higher than the competitive profits), in order to induce a firm to self-report, this firm will have to be compensated for foregoing its positive future expected profit. Since the current leniency programs in the OECD countries all feature $\alpha(\cdot) \geq 0$, the inevitable conclusion is that the ex-ante part of these programs is ineffective.

Second, (13) provides an upper bound on $\alpha(p)$ that depends only upon $\Lambda(p)$ and not on $\lambda(p)$. Therefore, this bound is indirectly related to the current antitrust regulation and some specific industry characteristics, like $\delta$ and $\gamma$, but is independent of the industry characteristic $\lambda(p)$, which may be the most difficult to estimate from empirical data.

Third, by the monotonicity of Lemma 11, it is easy to design an effective constant ex-ante leniency program such that (13) holds for all $p \in\left(p^{N}, p^{R}\right]$ :

$$
\alpha(p)=1-\frac{1}{\Lambda\left(p^{N}\right)}=\inf _{p \in\left(p^{N}, p^{R}\right]}\left[1-\frac{1}{\Lambda(p)}\right] \leq 1-\frac{1}{\Lambda(p)} .
$$

Although constant leniency program can be easily implemented in guidelines, it has the drawback of offering too much rewards for all $p>p^{N}$.

Fourth, legal and economic principles often differ and this is also the case for leniency programs. Since effective programs require rewards, this obviously violates the "crime should not pay" principle. Lemma 11, however, offers some scope for implementing reward schemes that reward less the more severe violations. This indicates a limited degree of moral or juridical reasons that can be allowed in effective ex-ante leniency programs. This result holds 
independently of the industry characteristics $\lambda(p)$, cartel culture $\gamma$, the discount factor $\delta$, and for any antitrust regulation as specified in Section 3.

Another economic principle is to keep the effective reward $\alpha(\cdot)$ as low as possible. Denote the minimal effective reward as $\hat{\alpha}(p)$, then after substituting (5) into (13), we obtain

$$
\alpha(p)<\hat{\alpha}(p)=\frac{\beta(p) k(p)-\delta[1-\gamma \beta(p)]}{1-\delta[1-\gamma \beta(p)]} .
$$

By definition, the minimal effective reward $\hat{\alpha}(p)$ has the properties stated in Lemma 11. The fact that cartels that set higher prices arouse more suspicions makes them more vulnerable to be uncovered. This implies a positive effect on the expected penalty and a negative effect on the expected time of enjoying the benefits from the cartel before the first conviction. Lower expected net benefits makes coming forward more attractive and, since the minimal effective reward is non-decreasing, requires a lower minimal effective reward. So, the presence of suspicions has a dampening effect on the minimal effective reward. Consequently, cartels that set low prices and by doing so arouse less suspicions are the most costly to terminate through leniency.

We now summarize our main findings in the following proposition:

Proposition 12 There always exists an effective ex-ante leniency program $\alpha(p)$ that satisfies (13) for all $p \in\left(p^{N}, p^{R}\right]$. This program eradicates all cartel prices in $\left(p^{N}, p^{R}\right]$, it requires rewards, i.e., $\alpha(p)<0$, and allows for a non-decreasing minimal reward leniency program $\hat{\alpha}(p)$.

Below we provide several remarks with respect to policy intervention and explain the main policy implications that follow from our results. First, it is important to realize that the minimal effective reward $\hat{\alpha}(\cdot)$ critically depends upon the existing traditional antitrust policy. So, changing the latter always has repercussions on the minimal reward ex-ante effective leniency program. Therefore, future reforms of the antitrust policy should consider simultaneously the traditional antitrust policy and the ex-ante leniency program. Second, the minimal effective reward has to be tailor-made to each industry or economic sector. 
Therefore, an one-size-fits-all ex-ante leniency program that by definition cannot incorporate industry characteristics and sector-dependent antitrust regulation inevitably either fails the minimal reward property in (14), or fails to be effective for some sectors and some cartel prices. In particular, constant ex-ante leniency programs for all sectors will involve too much rewarding firms at some cartel prices for some sectors and, where it remains ineffective, will offer too little incentives to come forward in other sectors. Third, since cartels that set low prices arouse little suspicions these cartels are the most costly to upset through tailor-made minimal-reward leniency programs. Such cartels, however, are also the cartels that cause the least damage to society. The trade-off between eliminating low-price cartels and the high costs through minimal rewards lies outside the scope of the current paper, but is certainly relevant for practice.

Our result that ex-ante leniency programs are ineffective generalizes the findings reported in Motta and Polo (2003), Spagnolo (2004) and Rey (2003) for very specific settings. In the last reference, it is also shown that the minimal effective reward is unbounded if $\delta$ goes to 1. Taking this limit for $\hat{\alpha}($.$) evaluated at arbitrary p$ in $\left(p^{N}, p^{M}\right]$ shows that this limit is bounded for all $\gamma>0$ and that it is only unbounded for the boundary case $\gamma=0$, which is the case analyzed in Rey (2003). Our results also hint at that the ineffectiveness of exante leniency programs without rewards reported in Harrington and Chen (2006) for the profit-maximizing cartel price under very large discount factors generalizes to a wider class of different objective functions for the cartel and all discount factors.

We now conclude this section by revisiting our example of the homogenous Bertrand oligopoly, which is also studied in Harrington and Chen (2006).

Example 13 Reconsider Example 8. Under antitrust regulation $\beta(p)=\beta p$ and $k(p)=k$, where $0<k \beta<1$, we first note that $\alpha(p)$ satisfies (13) for all $p \in\left(p^{N}, p^{M}\right]$ if and only if

$$
\alpha(p)<\hat{\alpha}(p) \equiv \frac{\beta k p-\delta(1-\gamma \beta p)}{1-\delta(1-\gamma \beta p)} .
$$

Since $\beta^{\prime}(p)=\beta>0$, this function is strictly increasing in $p$. The following figure plots 
several minimal reward leniency programs $\hat{\alpha}(p)$ for $\beta=0.2, k=3, \gamma=0.5$ and $\delta=0.7$, $\delta=0.8$ and $\delta=0.9$. The higher $\delta$, the larger the size of the minimal effective reward (in absolute value) needs to be.

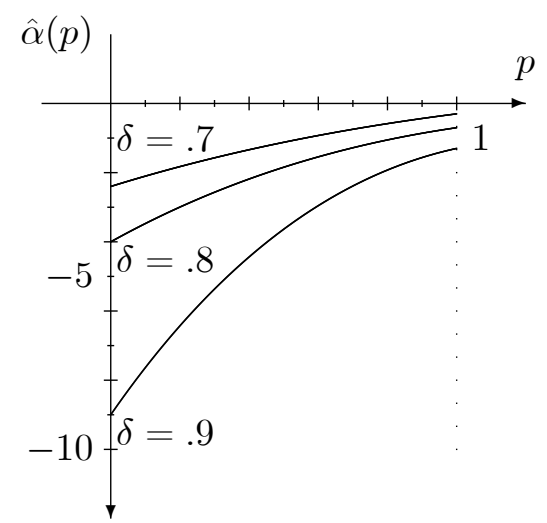

This figure illustrates that $\hat{\alpha}(p)$ is increasing in $p$, as asserted. When the actual leniency program $\alpha(p)$ is non-increasing in $p, \alpha(p)$ and $\hat{\alpha}(p)$ intersect at most once at $\tilde{p} \in\left(p^{N}, p^{M}\right]$ (in this last figure, which is equivalent to $(1-\alpha(p))^{-1}$ intersects $\Lambda(p)$ at most once at $\left.\tilde{p}\right)$. As the curve $\alpha(p)$ shifts downwards, i.e., the reward for all $p \in\left(p^{N}, p^{M}\right]$ increases, $p^{L}$ may decrease since the intersection point $\tilde{p}$ either moves to the left or stays the same. Finally, the following figure illustrates how the constant leniency program $\alpha(p)=-4$ affects the maximal cartel price under the benchmark, antitrust policy and effective leniency for the parameter values $\beta=0.2, k=3, \gamma=0.5$ above and $n=4$.

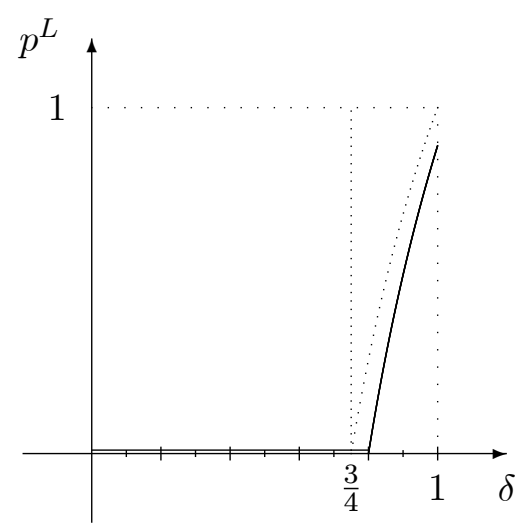

The vertical dotted line at $\delta=\frac{n-1}{n}=.75$ illustrates the discontinuous jump in the cartel 
price for the benchmark case (Example 2) and the dotted curve depicts the cartel price under antitrust regulation (Example 8). Note that $p^{R}=1$ at $\delta=1$ belongs to both possible cases illustrated in Example 8. The solid curve corresponds to $p^{L}$. It eradicates all cartel prices for $.75 \leq \delta \leq .8$ and reduces the maximal cartel price $p^{L}=\tilde{p}<p^{R}$ for $\delta>0.8$. Note that we cannot support $p^{L}=1$ because by (12) it would require $\delta \geq \Gamma(1)>1$.

\section{Concluding remarks}

In this paper, we explore a general infinitely-repeated game framework for the analysis of antitrust violations in the presence of both traditional antitrust policies and ex-ante leniency programs. This framework allows for the simultaneous analysis of two important decisions cartel members face in the presence of leniency programs: decisions to deviate from the cartel price and decisions to self-report to the authorities. A novel concept is the maximal cartel price that reflects society's worst cartel price among those cartel prices that are sustainable, which endogenizes the cartel formation decision and its pricing strategy. The generality of our framework is also reflected in allowing for endogenous detection probabilities and penalty schemes that are each non-decreasing in the cartel price. ${ }^{20}$ As a consequence, the expected punishment is also endogenous. Our framework allows to integrate decisions about self-reporting, cartel formation and its pricing strategy (maximal cartel price) and relate these to the type and structure of the industry including its cartel culture parameter.

Our analysis makes several contributions to the literature: A general technique to characterize and also to separate the effects of traditional antitrust enforcement and ex-ante leniency programs on the maximal cartel price. We also clarify that an important implication of the one-stage-deviation principle is that one can restrict any equilibrium analysis to either price deviations or deviations considering self-reporting, but it is unnecessary to consider both simultaneously.

\footnotetext{
${ }^{20}$ Endogenous detection probabilities offer a better description of real life situations and, therefore, are conceptually better suited for the analysis of antitrust violations. Instead, most of the literature on leniency assumes a fixed exogenous detection probability, which avoids the technical difficulties tackled in our paper.
} 
In this framework, we identify the sufficient and necessary conditions for which antitrust regulation is effective in reducing the maximal cartel price. We conclude that an antitrust policy is less effective in sectors where the degree of cartel stability is higher or where the sector's cartel culture to continue business as usual is more prominent. Confronting our theoretical results with stylized facts from currently employed antitrust policies in OECD countries imply that these policies are ineffective.

An effective ex-ante leniency program that eradicates all cartel prices always exists. Such leniency program, however, necessarily involves rewards. Furthermore, many reward schemes, including constant schemes, imply either too much or too little rewarding at some cartel prices. Since current leniency programs apply uniformly over all sectors, over/under rewarding will also differ across sectors. This implies that sector-specific minimal effective reward schemes would be the best solution in terms of offering precisely the amount of money to induce self-reporting.

The main results also have several policy implications. First, effectiveness of antitrust regulation and ex-ante leniency programs depends upon industry characteristics, such as industry or product life-cycle, sector's cartel culture, or the type of competition. Second, a minimal-reward leniency program has to be tailor made to each industry or economic sector instead of an one-size-fits-all leniency program. The reason is that an one-size-fits-all program may involve too much rewarding firms at some cartel prices in some economic sectors and may offer too little incentives to come forward in other sectors. Third, according to Rey (2003), AAs often do not observe relevant information in the absence of an audit. The presence of suspicions could be a reason for the authorities to develop antenna's for picking up such signals and develop filters to sift false rumors from true ones prior to an investigation. We do not investigate the costs and benefits of developing such policy instrument, but our analysis shows that if the authorities can transform suspicions into an effective policy instrument it will have a dampening effect on the maximal cartel price.

Our analysis also reveals that the profit-maximizing cartel price in Harrington (2004 and 
2005) may underestimate the maximal damage to society whenever the equilibrium conditions are nonbinding. As motivated in the introduction, economic agents often behave differently from standard microeconomic theory and the criterion of sustainability of cartel behavior offers an alternative and more robust framework that does not depend on the precise assumptions about cartel behavior. In this perspective, the maximal cartel price characterizes society's maximal damage and can therefore be regarded as a worst-case scenario.

The US Department of Justice (D.O.J.) reports empirical evidence in favor of the major modifications of its leniency program in 1993, see D.O.J. (1998). ${ }^{21}$ Despite this empirical evidence, Spagnolo (2007) asserts that leniency programs are still not fully understood theoretically. In particular, he raises the issue whether the observed increases in cartel detection are the result of unobserved increases in cartel activity (under equal detection probabilities) or are the result of improved effectiveness of leniency programs. Based on our results we are also able to address the last issue. Under a fixed traditional antitrust policy, introducing an ex-ante leniency program does not increase (unobserved) cartel activity. The ex-ante leniency programs in the US and the EC, however, are ineffective. Only sufficiently large rewards can increase cartel detection through revelation by the cartel. This is a general and robust result that goes far beyond the special cases studied in Spagnolo (2004), Rey (2003) and Harrington and Chen (2006).

An important goal set by theoretical articles, see e.g. the survey in Spagnolo (2007), is the determination of the optimal design of the antitrust policy and the leniency program. Studying the optimal design requires a well-defined framework for analyzing the effects of changes in the antitrust policy and the leniency program on society's welfare. Such changes can be thought of as shaping the functions describing the antitrust policy and the leniency program and, ideally, one would like a flexible and large class of such policy functions that

\footnotetext{
${ }^{21}$ Three major modifications were implemented: Amnesty is automatic if there is no pre-existing investigation; even if an investigation is under way, amnesty may still be available provided substantial evidence is brought forward; and all officers, directors, and employees who collaborate with the AA are protected from criminal prosecution.
} 
are a priori neither constant or linear. Although our analysis did not address optimal design directly, our framework allows for such a rich class of potential policy functions and a characterization of the maximal cartel price. Taking the latter price as a proxy for society's maximal damage, one can easily extend our framework by specifying social welfare as a function of the maximal cartel price and including society's costs of implementing certain policy functions. So, potentially, our framework could enhance the analysis of the optimal design of antitrust policies and leniency programs.

\section{References}

Abreu, D., D. Pearce, and E. Stacchetti (1986). Optimal cartel equilibria with imperfect monitoring. Journal of Economic Theory 39, 251-269.

Baumol, W. (1958). On the theory of oligopoly. Economica 25, 187-198.

Bosch, J. and E. Eckard (1991). The profitability of price-fixing: Evidence from stock market reaction to federal indictments. Review of Economics and Statistics 73, 309317.

Buccirossi, P. and G. Spagnolo (2006). Leniency programs and illegal transactions. Journal of Public Economics 90(6-7), 1281-1297.

D.O.J. (1993). Us corporate leniency policy. http://www.usdoj.gov/atr/public/guidelines/0091.htm.

D.O.J. (1998). The twelfth annual report of the department of justice. http://www.usdoj.gov/atr/public/speeches/1583.html.

EC (2002). Commission adopts new leniency policy for companies which give information on cartels. Brussels, http://europa.eu.int/comm/competition/antitrust/leniency/.

Fudenberg, D. and J. Tirole (1991). Game Theory. MIT Press, Cambridge.

Green, E. and R. Porter (1984). Noncooperative collusion under imperfect price information. Econometrica 52, 87-100. 
Harrington, J. (2004). Cartel pricing dynamics in the presence of an antitrust authority. The Rand Journal of Economics 35, 651-673.

Harrington, J. (2005). Optimal cartel pricing in the presence of an antitrust authority. International Economic Review 46, 145-170.

Harrington, J. and J. Chen (2006). Cartel pricing dynamics with cost variability and endogenous buyer detection. The International Journal of Industrial Organization 24(6), $1185-1212$.

Hinloopen, J. (2003). An economic analysis of leniency programs in antitrust law. De Economist 151, 415-432.

Hinloopen, J. (2006). Internal cartel stability with time-dependent detection probabilities. The International Journal of Industrial Organization 24(6), 1213-1229.

McGuire, J., J. Chiu, and A. Elbing (1962). Executive incomes, sales and profits. American Economic Review 52, 753-761.

Motchenkova, E. (2004). Effects of leniency programs on cartel stability. CentER Discussion Papers Series 2004-98, Tilburg University, Tilburg.

Motta, M. and M. Polo (2003). Leniency programs and cartel prosecution. International Journal of Industrial Organization 21, 347-379.

OECD (2002). Fighting hard-core cartels: Harm, effective sanctions and leniency programs. OECD Report 2002, OECD, Paris, France, http://www.SourceOECD.org.

Polinsky, M. and S. Shavell (2000). The economic theory of public enforcement of law. Journal of Economic Literature XXXVIII, 45-76.

Rey, P. (2003). Towards a theory of competition policy. included in the book "Advances in Economics and econometrics" series of the econometric society, published in 2003.

Spagnolo, G. (2004). Optimal leniency programs. Centre for Economic Policy Research, Discussion paper series, 4840, http://www.cepr.org/pubs/new- 
dps/showdp.asp?dpno $=4840$.

Spagnolo, G. (2007). Fines, leniency and whistleblowers in antitrust. forthcoming as Ch. 12 of P. Buccirossi (Ed.),Handbook of Antitrust Economics, 2007, M.I.T. Press, http://www.gianca.org/PapersHomepage/Spagnoloblowers.pdf.

Wen, Q. (2002). A folk theorem for repeated sequential games. Review of Economic Studies 69, 493-512. 